\title{
High Performance, Low Cost, Colorless ONU for WDM-PON
}

\author{
Ryohei Urata, Cedric Lam, Hong Liu, and Chris Johnson \\ Google Inc., 1600 Amphitheatre Parkway, Mountain View, CA 94043, USA \\ ryohei@google.com
}

\begin{abstract}
We give an overview of key technologies for realizing WDM-PON. In particular, we highlight promising developments and directions in widely tunable laser technologies for achieving a high performance, colorless ONU at the cost points required for access networks. OCIS codes: (060.2330) Fiber optics communications; (060.4250) Networks; (140.3600) Lasers, tunable
\end{abstract}

\section{Introduction}

Over the past several years, end-user access bandwidth has grown exponentially throughout the world as a result of the rollout of broadband networks such as fiber-to-the-home (FTTH). This increased bandwidth has enabled a variety of new web-based applications, cloud computing, and content streaming. Continued enhancement of these services, along with new applications, are expected to further drive the demand for bandwidth.

This growth of access bandwidth has, in part, been supported by various initiatives, with the underlying belief that broadband connectivity and infrastructure boosts creativity and productivity. To this end, Google has embarked on a FTTH program with the intent to provide and boost broadband access speeds to 1-Gb/s connections in select locations throughout the United States [1]. An initial trial network has been constructed at Stanford University, with the first Google Fiber markets planned in Kansas City, Kansas and Kansas City, Missouri.

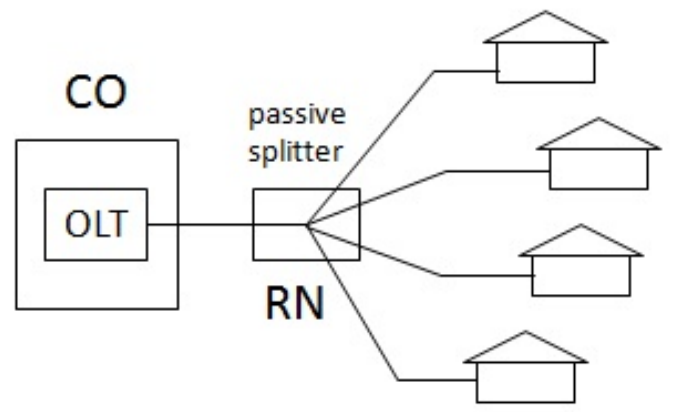

(a) TDM-PON

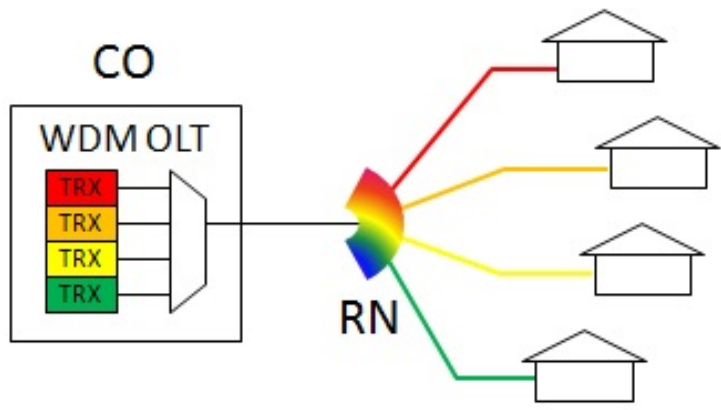

(b) WDM-PON

Fig. 1: Simple illustrations of a) TDM-PON and b) WDM-PON.

Up to this point, FTTH deployments have predominantly utilized a time division multiplexed (TDM)-passive optical network (PON) scheme due to simplicity of implementation and the ability of technologies to easily support the desired data rates at the time of adoption. Illustrated in Fig. 1a), a shared optical line terminal (OLT) transceiver at the central office (CO) broadcasts downstream to individual optical network units (ONUs), where the correct packets are selected out as part of the PON protocol. In the reverse, upstream direction, transmission occurs in a burst mode fashion to allow time sharing of the single communication channel. However, as the need for bandwidth increases, it is clear that this traditional PON architecture will not be able to scale, as all transceivers must work at the aggregate bit rate of the entire system. In addition, the unique protocol required for TDM-PON to function does not allow end-to-end Ethernet protocol transparency, with a cumbersome conversion needed from TDM-PON protocol to Ethernet (and back) at all end points.

WDM-PON, using wavelength division multiplexing (WDM), has long been touted as the key to delivering symmetric and sustained bandwidth at or beyond $1 \mathrm{~Gb} / \mathrm{s}$ to each user. Higher security can also be an added benefit. Over the years, a variety of implementations and corresponding device technologies have been investigated as a result [2]. In this paper, we give an overview of key technologies for achieving a high performance, robust WDMPON. In particular, we highlight promising developments and directions in tunable laser technologies for enabling a 
high performance, colorless ONU with low power consumption and the cost points required for adoption in access networks.

\section{WDM-PON System}

Fig. 1b) illustrates the WDM PON system. The CO contains multiple transceivers at different wavelengths with each output wavelength creating a dedicated path or channel for a particular user by passing through a wavelength selective/dependent element at the remote node (RN). Wavelength selection can also be achieved by filtering at the user. The upstream connection similarly utilizes a dedicated wavelength channel. With this architecture, OLT and ONU transceivers need only operate at the data rate directly seen by the user, and the Ethernet protocol is preserved from end-to-end. However, these advantages over TDM-PON are accompanied by challenges in realizing the transceivers at the end points, as well as the remote node.

For the WDM-PON remote node, there have been significant advancements in athermal arrayed waveguide grating (AWG) technology [3]. This has paved the way for a compact, monolithic remote node which exhibits good performance over temperature hardened conditions $\left(-40\right.$ to $\left.+85^{\circ} \mathrm{C}\right)$, with products being commercially available in the market today. High delta waveguides allow tight bends, leading to a compact cartridge size comparable to that for a splitter.

At the OLT, adopting individual transceiver units for each WDM channel increases size, cost, and power. Thus, for the CO, the integration of the transmit, receive, demux, and mux components into an integrated WDM transceiver is critical, achieved through either monolithic integration and/or novel packaging techniques [4].

\section{Colorless ONU}

Perhaps the most challenging component to achieve is the low cost ONU transceiver, which must be colorless. A colorless ONU supporting all wavelength channels is needed in order to ease operation and maintenance and reduce cost through volume production of one component. A variety of creative schemes and devices have been investigated. The first is the use of a broadband light source (BLS) as the transmitter, with the output signal of the BLS being spectrally sliced by the remote node. However, there are major issues hindering performance, namely power loss through the remote node, dispersion, and spontaneous-spontaneous beat noise limitation. Reflective semiconductor optical amplifiers (RSOAs) have been studied intensively, with progress made in reducing reflection of the end facet, increasing modulation speed, and achieving polarization insensitive operation [5]. However, the bottlenecks have been the high amount of injected power required, inherently poor signal quality, and dispersion when using a broadband seeding method or complexity and difficulty of implementation when using a laser seeding method. In effect, any laser seeding scheme requires a second set of WDM sources at the CO. Systems incorporating injection-locked Fabry-Perot lasers as the ONU transmitter also can be divided into broadband seeding and laser seeding approaches [6]. The former requires a high amount of power to lock the laser sufficiently, and the latter is polarization dependent, requiring polarization control or diversity which will add a significant amount of complexity and cost to the transceiver. Finally, with all seeding methods, signal quality is highly sensitive to reflections throughout the fiber plant.

From a performance standpoint, the availability of an ONU with a wavelength tunable laser will provide superior signal quality, reach, and stability, with the wavelength of each laser tuned to the appropriate upstream channel. The underlying issue has been the cost of the widely tunable lasers being unable to reach the cost points required for adoption in FTTH, being roughly an order of magnitude too expensive.

With the idea that photonic integration could be leveraged to reduce size, cost, and power and increase functionality, there has been considerable development of distributed Bragg reflector (DBR)-type tunable lasers [79]. However, the complicated fabrication which requires electron beam or high resolution lithography for defining the gratings and in particular, the need to bury the gratings with a regrowth step, can both increase chip cost and impact yield. In addition, size of the laser inevitably becomes larger with the DBR sections.

With this in mind, in recent years, work has been aimed at realizing tunable lasers with a simplified structure and fabrication process. Shown in Fig. 2a), NTT has recently developed a double-ring-resonator-coupled tunable laser [10]. The tunable elements are a pair of ring resonators, which eliminate the need for gratings. The compact size of the rings also helps to reduce chip size. Their superior filter characteristics lead to low tuning currents to reduce power consumption. Shown in Fig. 2b), NEC has taken the approach of simplifying the required III-V semiconductor block further, with only the gain block being fabricated in InP-based material and the ring resonator tuning elements realized in a silicon photonics platform [11]. Heating of the rings induces a thermo-optic effect which shifts the wavelength peak of the rings. The independent optimization and high yield of the various 
components is at the expense of packaging complexity and the need to achieve good optical alignment and reduce reflections within the laser cavity.

In addition to these techniques for reducing size, power, and cost, remaining challenges are maintaining stable lasing without the use of a wavelength locker as well as reducing the required testing time to increase manufacturing throughput [9].

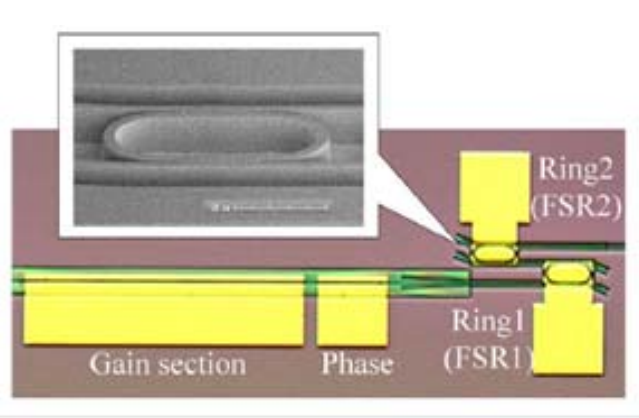

a)

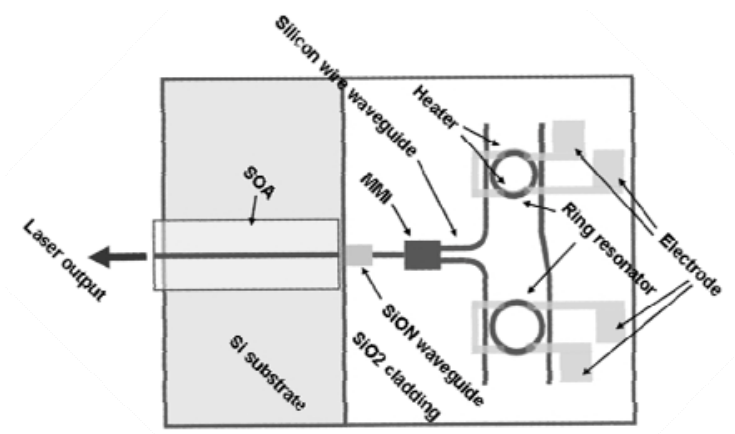

b)

Fig. 2: a) Double-ring-resonator coupled tunable laser (courtesy of [10]). b) Tunable laser with silicon photonic micro-ring resonators (courtesy of [11]).

\section{Conclusion}

WDM-PON is the most promising long-term, scalable solution for delivering high bandwidth to the end user. Progress in key device technologies has laid the foundation for realization of a high performance, low cost WDMPON system, although challenges remain. These advances will be needed to not only scale to $1 \mathrm{~Gb} / \mathrm{s}$, but to $10 \mathrm{~Gb} / \mathrm{s}$ per user and beyond.

\section{References}

[1] C.F. Lam, “The road to scalable 1Gb/s FTTH access networks,” ECOC 2011, Tu6.C.2.

[2] J. Kani, “Enabling technologies for future scalable and flexible WDM-PON and WDM/TDM-PON systems,” IEEE J. Sel. Topics Quantum Electron., vol. 16, no. 5, Sept./Oct. 2010, pp. 1290-1297.

[3] S. Kamei, "Recent progress on athermal AWG wavelength multiplexer,” OFC/NFOEC 2009 , OWO1.

[4] H. Liu, C.F. Lam, and C. Johnson, “Scaling optical interconnects in datacenter networks,” IEEE Symposium on High Performance Interconnects 2010.

[5] F. Payoux, P. Chanclou, and R. Brenot, “WDM PON with a single SLED seeding colorless RSOA-based OLT and ONUs,” ECOC 2006, Tu4.5.1.

[6] S. Park, C. Lee, K. Jeong, H. Park, J. Ahn, and K. Song, "Fiber-to-the-home services based on wavelength-division-multiplexing passive optical network,” IEEE J. Lightw. Technol., vol. 22, no. 11, pp. 2582-2591, Nov. 2004.

[7] V. Jayaraman, Z.-M. Chuang, and L.A. Coldren, "Theory, design, and performance of extended tuning range semiconductor lasers with sampled gratings,” IEEE J. Quantum Electron., vol. 29, no. 6, pp. 1824-1834, Jun. 1993.

[8] H. Ishii, Y. Tohmori, Y. Yoshikuni. T. Tamamura, and Y. Kondo, "Multiple-phase shift super structure grating DBR lasers for broad wavelength tuning,” IEEE Photon. Technol. Lett., vol. 5, no. 6, pp. 613-615, Jun. 1993.

[9] A. C. Carter, “Evolution of optical component technologies for access and metro networks,” ECOC 2009, Paper 9.5.1.

[10] S. Matsuo and T. Segawa, "Microring-resonator-based widely tunable lasers,” IEEE J. Sel. Topics Quantum Electron., vol. 15, no. 13, May/June 2009, pp. 545-554.

[11] N. Fujioka, T. Chu, and M. Ishizaka, "Compact and low power consumption hybrid integrated wavelength tunable laser module using silicon waveguide resonators,” IEEE J. Lightw. Technol., vol. 28, no. 21, pp. 3115-3120, Nov. 2010. 